\title{
Target-Decoy MineR for determining the biological relevance of variables in noisy data sets.
}

\author{
Cesaré Ovando-Vázquez ${ }^{1,2 *}$, Daniel Cázarez-García ${ }^{2}$ and Robert Winkler ${ }^{2 * *}$
}

\begin{abstract} https://bitbucket.org/cesaremov/targetdecoy_mining/.

Keywords

data mining, Drosophila, metabolomics, bioinformatics, statistics

${ }^{1}$ Potosinan Institute for Scientific and Technological Research (IPICYT), San Luis Potosí, Mexico

${ }^{2}$ Center for Research and Advanced Studies (CINVESTAV), Irapuato, Mexico

Corresponding authors: *cesaremov@gmail.com, **robert.winkler@cinvestav.mx
\end{abstract}

Machine learning algorithms excavate important variables from biological big data. However, deciding on the biological relevance of identified variables is challenging. The addition of artificial noise, 'decoy' variables, to raw data, 'target' variables, enables calculating a false-positive rate (FPR) and a biological relevance probability (BRp) for each variable rank. These scores allow the setting of a cut-off for informative variables can be defined, depending on the required sensitivity/ specificity of a scientific question. We demonstrate the function of the Target-Decoy MineR (TDM) with synthetic data and with experimental metabolomics results. The Target-Decoy MineR is suitable for different types of quantitative data in tabular format. An implementation of the algorithm in $R$ is freely available from

\section{Contents}

1 Introduction

\section{1}

1.1 Machine learning, variable importance scoring problem 2

\section{Methods}

2.1 Target-Decoy MineR algorithm . . . . . . . . . 2

2.2 Synthetic data . . . . . . . . . . . . . 4

2.3 Origin and cultivation of Drosophila samples ... . 4

2.4 Sample preparation ... . . . . . . . . . . . 4

2.5 Non-targeted metabolic profiling . . . . . . . . 4

2.6 Experimental data processing . . . . . . . . 4

2.7 Target-decoy table generation . . . . . . . . . 4

2.8 Univariate analysis . . . . . . . . . . . . . 4

2.9 Variable importance scores, multivariate analysis . . 5

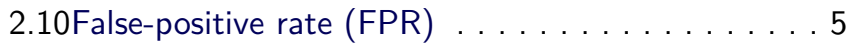

2.11Target and decoy class definition ........ 5

2.12Biological relevance probability (BRp) ...... 5

2.13Supervised principal components (sPC) ... . . 5

2.14Pre-conditioning of data . . . . . . . . . . 5

2.15Root mean square error (RMSE) . . . . . . . . 5

2.16 Cross-validation ............... . 5

2.17Receiver operating curve $(\mathrm{ROC})$ analysis .... . 5

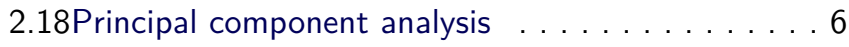

2.19Data availability statement . . . . . . . . . 6
3 Results

3.1 Processing synthetic datasets with defined perturbations 6

3.2 Processing experimental metabolomics data . . . . 6

3.3 Statistical performance of the Target-Decoy MineR 6

3.4 Robustness of the Target-Decoy MineR against noise 9

4 Discussion 9

5 Conclusion 9

6 Acknowledgments 9

7 Author contributions 9

8 Competing financial interests 9

\section{Introduction}

Extracting informative variables from a noisy background is necessary to convert massive data sets into biological models. Statistical analyses, such as the generalized linear model for non-normal data, are used to extract these relevant variables. However, setting a stringent cut-off for statistical significance leaves fewer variables for interpretation. This sensitivity-specificity dilemma (SSD) is accompanied by the difficulty to rank variables according to their biological relevance. 
bioRxiv preprint doi: https://doi.org/10.1101/2020.11.09.374181; this version posted November $10,2020$. The copyright holder for this preprint (which was not certified by peer review) is the author/funder, who has granted bioRxiv a license to display the preprint in perpetuity. It is made available under aCC-BY-NC-ND 4.0 International license.

Target-Decoy MineR for determining the biological relevance of variables in noisy data sets. $-2 / 11$

\subsection{Machine learning, variable importance scoring problem}

Supervised and unsupervised machine learning (ML) methods play a crucial role in dealing with the accelerated availability of big data in biology ${ }^{1}$. The random forest (RF) algorithm is an ML method that builds multiple decision tree models from randomly selected variables of a massive training data set and integrates the results into a final model ${ }^{2,3}$. The process is computationally efficient and robust against noise. The model builder estimates an out-of-bag (OOB) error, which indicates the reliability of the model for sample classification. Computed variable importance values designate each variable's contribution to the correct splitting in the decision trees ${ }^{4}$. As a measure, the overall decrease of diversity by the variable expressed as the mean decrease Gini (MDG) is used ${ }^{5,6}$. Since this calculation is unbiased by the data scientist's knowledge, RF models enable the discovery of unexpected relationships ${ }^{7}$. The $\mathrm{RF}$ algorithm frequently serves for the variable selection and classification of genes from microarray data ${ }^{8}$. In mass spectrometry-based Omics, which face the same problem, i.e., large numbers of variables and relatively few experimental repetitions, data mining (DM) strategies such as RF analysis demonstrated to be superior for sample classification to clustering algorithms ${ }^{9}$. Thus, DM strategies are routinely employed in bioinformatics for multiclass classification and variable selection ${ }^{10}$.

Despite all these RF advantages, RF lacks sparsity in the final models, representing a serious limitation when dealing with thousands of variables. Also, due to its intrinsic random nature, variable importance stability must be tested. Sparse partial least square discriminative analysis (sPLS-DA) is a supervised deterministic ML classification methodology performing variable selection ${ }^{10}$. sPLS-DA identifies a small subset of best variables contributing to discrimination between sample classes. This subset of best discriminative variables has a variable importance score, expressed as loadings. Each load indicates the contribution to the discriminative decision function and has a direct interpretation ${ }^{10}$. sPLS-DA has been used to investigate single nucleotide polymorphisms (SNP) and single nucleotide variants (SNV) data ${ }^{10,11}$.

Both RF and sPLS-DA are powerful tools for analyzing quantitative biological data. Nevertheless, the variable selection from RF and sPLS-DA analyses remains challenging ${ }^{12}$, particularly the direct interpretation of the variable importance score and setting an optimal cut-off for possibly relevant variables. One possibility for assessing the contribution of single variables (targets) is their permutation and subsequent statistical evaluation. This method performs better than previously described strategies but is computationally demanding ${ }^{13}$.
These ML models, RF, and sPLS-DA must be validated using an independent set to estimate their performance. Given the specificity and scarcity of metabolomic datasets, it is impossible to keep out an independent validation set and use it to get a test error $^{14}$. Cross-validation (CV) is a straightforward and widely used methodology to estimate ML models' prediction error ${ }^{14}$. $k$-fold cross-validation splits the data into $k$ subsets. The model is trained with $k-1$ subsets and validate it on the left out sub-set. Five- or ten-fold cross-validation is considered a good compromise between bias and variance ${ }^{15}$. However, it depends on the number of samples available in the dataset.

In proteomics, the target-decoy (TD) strategy is commonly used for estimating the confidence of protein identifications. Spectral matching is performed against a concatenated database, which consists of correct ('tar$g^{\prime} t^{\prime}$ ) and false ('decoy'), i.e., random protein sequences. Based on the proportion of (falsely) identified decoy proteins, a false positive rate (FPR) can be calculated, assuming that target and decoy hits occur with equal likelihood $^{16}$.

In this work, we combined the target-decoy strategy and machine learning techniques. We call this methodology the 'Target-Decoy MineR (TDM).'

\section{Methods}

\subsection{Target-Decoy MineR algorithm}

The Target-Decoy MineR builds a sample classification model, using a dataset of $S$ samples with $T$ variables (targets) (Fig. 1a). Decoys are generated from the targets, permuting the sample class, obtaining $D$ variables. Targets and decoys are gathered to obtain a TargetDecoy data matrix (Fig. 1b). The Target-Decoy MineR provides a ranking of variables according to the variable importance, false-positive rate, and biological relevance, using the decoys as variables that are unrelated to the sample class.

The variable importance rank for values in the TargetDecoy data matrix is obtained by combining random forest (RF) and sPLS-DA scores. Given the variable importance ranking, a supervised principal components (sPC) model (sample classification model) with the top-ranked $m$ variables is trained (Fig. 1c) ${ }^{14}$. The sPC model is generated using $C$ principal components $(C \leq S)$, as input variables. The optimum model with $m$ variables is obtained by varying the top-ranked variables. The minimum of the Target-Decoy MineR metric is located at the optimum variable ranking $r_{-} o p t$. At the r_opt, Target-Decoy MineR identifies the perturbated variables of an experiment (Fig. 1c), i.e., the biologically relevant variables which contribute to the differentiation of samples.

In parallel to sPC model training (Fig. 1c), the decoy variables enable the calculation of false-positive rates 
bioRxiv preprint doi: https://doi.org/10.1101/2020.11.09.374181; this version posted November 10,2020 . The copyright holder for this preprint (which was not certified by peer review) is the author/funder, who has granted bioRxiv a license to display the preprint in perpetuity. It is made available under aCC-BY-NC-ND 4.0 International license.

Target-Decoy MineR for determining the biological relevance of variables in noisy data sets. $-3 / 11$

\section{TDM algorithm}
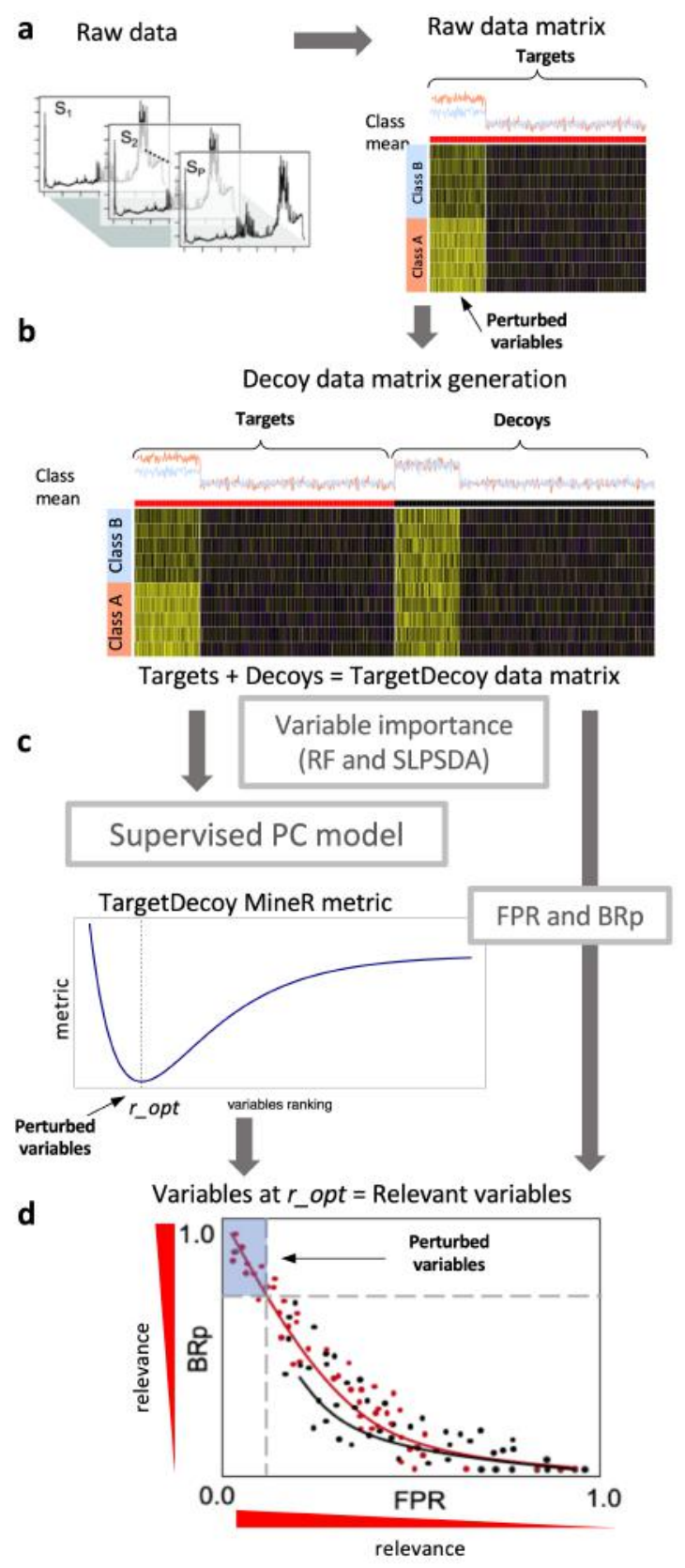

TDM on synthetic data
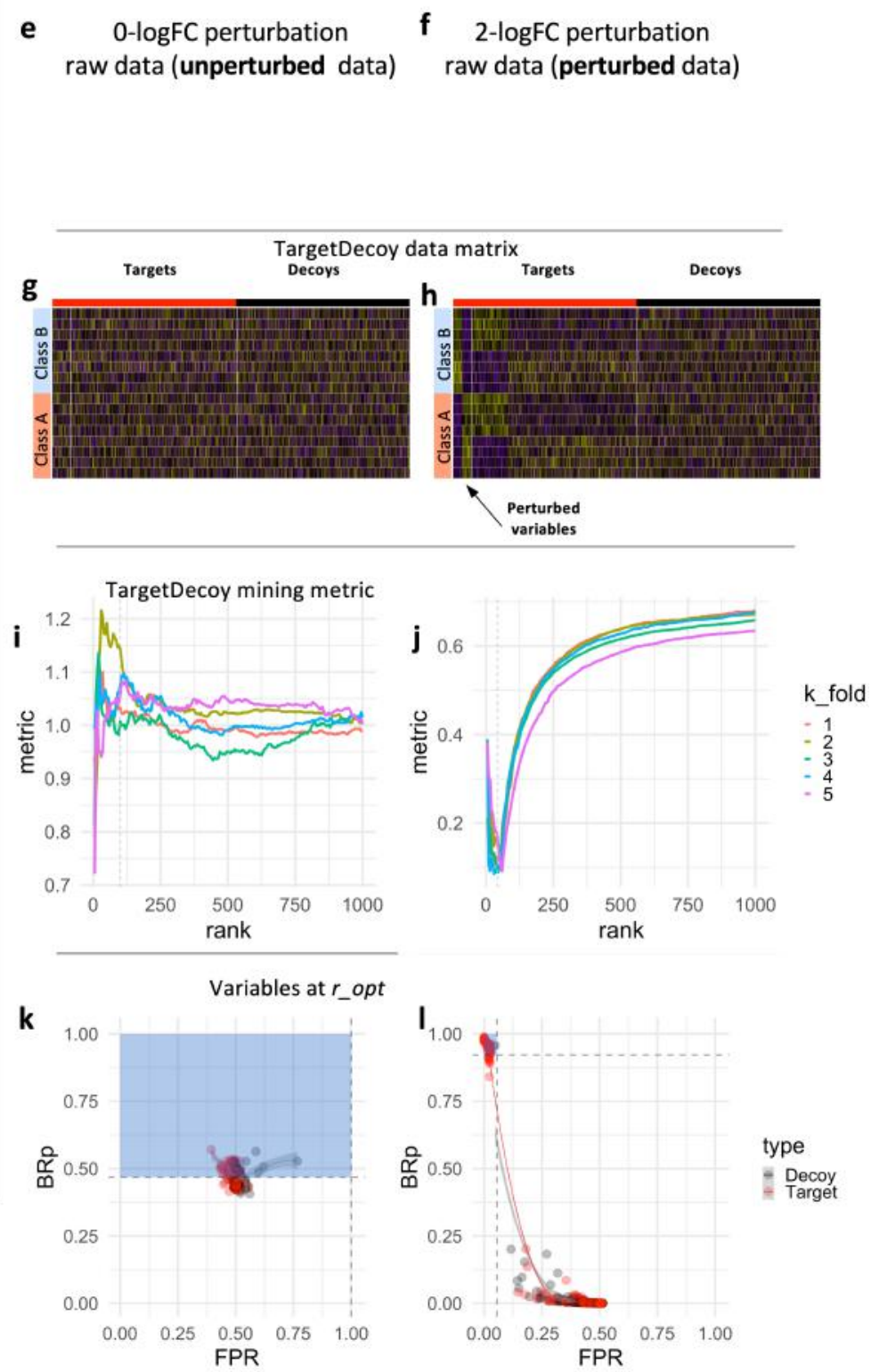

Figure 1. The Target-Decoy MineR (TDM) identifies biologically relevant variables in metabolomics datasets. a-d: Algorithm, a - Identification of variables in datasets representing two sample classes (A and B). $\mathbf{b}$ - Creation of a matrix with experimental (target) and artificial noise (decoy) data, c - Calculation of optimum metric and rank using a $k$-fold cross-validation, $\mathbf{d}$ - biological relevance probability (BRp) vs. false-positive rate (FPR) plot. e-l: Test with synthetic data. e-h synthetic data sets with $500 \mathrm{~m} / z$ variables; 50 variables are perturbed with 0 - and 1$\log$ FC. The perturbed variables correlate with the sample class, $\mathbf{g}, \mathbf{h}$ Target-decoy matrix, $\mathbf{i}-\mathbf{j}$ Calculation of the optimum metric and rank, using a 5-fold cross-validadion, k-1 BRp vs. FPR plots. For the 0-logFC dataset, no relevant variables were detected $(\mathbf{k})$; in the $1-\log F C$ dataset, variables contributing to the classification are ranked according to their relevance (1). 
(FPR). The FPR indicates the proportion of synthetic noise variables at a certain variable importance ranking. Besides the FPR, a biological relevance probability (BRp) is calculated, using the top target and top decoy variable importance scores. The BRp is the likelihood of a variable being truly related to a sample class.

During the variable importance calculation, sPC model training, FPR, and BRp calculation, $k$-fold crossvalidation $(\mathrm{CV})$ procedure is used; those validation stages prevent overfitting. In each fold, the TargetDecoy MineR trains the sPC model with all samples in the $k-1$ folds and validates it with the samples in the $k$ fold validation dataset. The variables in the validation dataset are pre-conditioned with the $\mathrm{BRp}^{14}$.

The Target-Decoy MineR dissects the dataset and accepts or denies changing variables. Further, the variable scores (FPR and BRp) and the workflow graphics enable a straight-forward visual evaluation of the model built from the input data. Thus, the TDM allows us to judge an experiment (Fig. 1d) quickly. Importantly, the Target-Decoy MineR model generation takes into account variable-variable relations.

\subsection{Synthetic data}

We used the Cardinal package ${ }^{17}$ to generate artificial Mass Spectrometry data using the function simulateSpectrum() with default parameters and $\mathrm{n}=5$, from $=50$, to $=1500$, baseline $=1$, by $=$ 100. The 5 spectra simulated were added up to obtain just only 1 spectrum. Then we used the $R$ package MALDIquant ${ }^{18}$ to process these spectra. First, we transformed spectra to mass spectrum profiles object using the function createMassSpectrum from the $\mathrm{R}$ package MALDIquant. Then, we used thebaseline, alignSpectra, detectPeaks, binPeaks with a tolerance $=0.1$, filterPeaks with minFrequency $=$ 0.5 , and intensityMatrix functions from MALDIquant to get a feature matrix (simulated metabolic data). We used this methodology to create a dataset with $n=10$ replicates and $m=500$ variables in wild type (WT) and treatment (TR) conditions. We perturbed only 50 variables, over-expressing 25 in WT and 25 in TR. The degree of perturbation was $\log F C=0,0.5,1,2$.

\subsection{Origin and cultivation of Drosophila samples}

We re-analyzed data reported by ${ }^{19}$. As briefly described below and in the following sections, we grew isofemale lines of Drosophila melanogaster and Drosophila mojavensis in standard banana and yeast food at $25^{\circ} \mathrm{C}$ and 12 hours light/dark. We put hundreds of flies in chambers with Petri dishes with $0.5 \%$ agar and active yeast and $5 \mathrm{~mL}$ of prickly pear for Drosophila mojavensis (to induce oviposition). We fed third instar larvae with a high protein-tosugar or equal protein-to-sugar ratio diet ${ }^{20}$. We sorted adult flies by sex at 0 days of eclosion.

\subsection{Sample preparation}

We lyophilized and weighted samples. We homogenized dry-samples with a polypropylene pestle and liquid nitrogen. We added $66 \mu \mathrm{L}$ of $85 \%$ methanol cold at $-20{ }^{\circ} \mathrm{C}$ per mg of dry mass. We put samples through 5 cycles of 1-minute ultrasound at $40 \mathrm{kHz}$ and 1 minute (on ice). We centrifuged samples at 15,870 g for $10 \mathrm{~min}$ utes at $4{ }^{\circ} \mathrm{C}$. We collected the supernatant for Drosophila melanogaster, and for Drosophila mojavensis, we filtered samples through a $0.2 \mu \mathrm{m}$ nylon syringe filter.

\subsection{Non-targeted metabolic profiling}

We analyzed extracts in random order for each species in distinct blocks. We used an Accela UPLC coupled to an LCQ Fleet Ion trap, using a Hypersil Gold C18 column $(50 \times 2.1 \mathrm{~mm}, 1.9 \mu \mathrm{m}$ particle size $)$ at $30{ }^{\circ} \mathrm{C}$ with a flow rate of $400 \mu \mathrm{L} / \mathrm{min}$. The mobile phase was water with $0.1 \%(v / v)$ formic acid and methanol with $0.1 \%$ $(v / v)$ formic acid. We acquired spectra in positive centroid mode in a range from 100 to $1,400 \mathrm{~m} / z$.

\subsection{Experimental data processing}

We analyzed experimental data with MZmine $2.21^{21}$ as reported in ${ }^{19}$. For feature detection, we used GridMass $^{22}$ with a minimum relative intensity of 100 . We normalized data with the maximum intensity peak. We used raw experimental data for TDM. We also removed features detected in blanks for the denoised data set before TDM.

\subsection{Target-decoy table generation}

Decoys were generated randomizing the target data (along rows and columns) using the function sample() from the $\mathrm{R}$ package base ${ }^{23}$ until finding a population of targets and decoys with no evidence favoring the hypothesis

$$
H_{0}: a b s\left(\rho_{\text {Target }}\right)=a b s\left(\rho_{\text {Decoy }}\right)
$$

(Wilcoxon signed rank test ${ }^{23}$ ) with a significance cut-off of

$$
p-\text { value }=0.01
$$

\subsection{Univariate analysis}

We used a generalized linear model (GLM) to test the hypothesis

$$
H o: \mu_{A j}=\mu_{B j}
$$

per $m / z$ variable (Target or Decoy) for biological conditions $A$ and $B$. We used glm.nb () from the R package MASS $^{24}$ with default parameters. False discovery rate (FDR) was performed with the Benjamini-Holchbech correction $^{25}$ function p.adjust() from the R package stats $^{23}$. 
2.9 Variable importance scores, multivariate analysis We combined two-variable importance scores from two machine learning methods to get the TDM variable's importance. These two machine learning methods are sparse partial least square discriminant analysis (SPLSDA) classifier and the random forest (RF). We developed a sparse partial least square discriminant analysis (SPLSDA) classifier model using conditions as classes and $m / z$ (target and decoy) variables as features. We generated the SPLSDA with the function splsda from the R package MixOmics ${ }^{10}$, using default parameters for both synthetic and Drosophila species datasets. From the SPLSDA model, we obtained the loadings as variable importance scores. We selected SPLSDA due to its sparsity, minimizing the score of nonrelevant variables. However, SPLSDA could develop low-complexity models. We developed the RF classifier models with the functionranger ()$^{26}$, using the default parameters for both synthetic and Drosophila species datasets. We used the mean decrease Gini (MDG) values as the variable importance score. We selected RF due to its balance between model bias and complexity. The TDM variable importance of targets and decoys is the average of the mean decrease Gini (MDG) and the sparse partial least square discriminant analysis (SPLSDA) loading scores ${ }^{14}$. Target and decoy variables were concatenated for calculating the target-decoy variable importance.

\subsection{False-positive rate (FPR)}

Variables were sorted in decreasing TD scores order and ranked using the rank () function from the $\mathrm{R}$ base package $^{23}$. We calculated FPR as the ratio of the number of decoys $|D|$ to the number of decoys and targets $|D \cup T|$ at rank $r$ :

$$
F P R_{r}=\frac{|D|_{r}}{|D \cup T|_{r}}
$$

\subsection{Target and decoy class definition}

We used the top target and decoy variables (variables between the 90 and 100 percentiles) as the representative class definitions.

\subsection{Biological relevance probability (BRp)}

A support vector machine (SVM) classification model ${ }^{14}$ was trained using the top-ranked target and decoy variables (variables between the 90 and 100 percentiles). We used the data from the class definition mentioned previously to train the model. The SVM model delivers probabilities for all target and decoy variables, representing the biological relevance probability (BRp). A linear classification model was generated using the function svm from the R packagee $1071^{27}$ with default parameters with linear kernel, and classification type ' $C$ classification.'

\subsection{Supervised principal components (sPC)}

Top-ranked variables $m$ were used to build a Principal Component Analysis (PCA) model using the $\mathrm{R}$ functionprcomp ${ }^{23}$. We used the $C, C=\min (2$, nrow(testData)), principal component (PC) variables to reduce train and test data to $C$ variables. We fitted a linear model on the C PC space using the function $\mathrm{lm}$ from the $\mathrm{R}$ package stats $^{23}$. The trained linear model was used to predict the class output from reduced PC validation data.

\subsection{Pre-conditioning of data}

Each variable in the validation dataset is multiplied by its corresponding BRp score, preconditioning TD variables in the validation dataset.

\subsection{Root mean square error (RMSE)}

We used the root mean square error (RMSE) to measure the sPC model prediction accuracy. We obtained validation predictions using the trained linear model and compared it to the actual validation sample class with a custom $R$ function ${ }^{23}$ :

$$
R M S E=\sqrt{E\left((y-\hat{y})^{2}\right)}
$$

, where $y$ is the sample class model prediction, and

$$
\hat{y}
$$

is the real sample class.

Metric. We defined the metric to optimize as the ratio of RMSE of predictions from the model built upon top $m$ ranked variables to predictions from the model using the rest of variables $(n)$ with a custom $\mathrm{R}$ function ${ }^{23}$. The total number of variables are total $=m+n$. The TDM metric is defined as:

$$
\text { TDMmetric }=\frac{R M S E_{m}}{R M S E_{n}}
$$

\subsection{Cross-validation}

We performed $k$-fold cross-validation using one out of $k$ samples to get target-decoy scores, FPR, and BRp values. We found the optimum FPR and BRp scores in each fold, minimizing the above-mentioned metric using the $k$ fold data.

\subsection{Receiver operating curve (ROC) analysis}

We used ROC analysis and Area Under the Curve $(\mathrm{AUC})^{28}$ to evaluate the global performance: sensitivity and specificity. We use the function $\operatorname{roc}()$ from the $\mathrm{R}$ package $p R O C^{29}$ to calculate AUC values. 
bioRxiv preprint doi: https://doi.org/10.1101/2020.11.09.374181; this version posted November $10,2020$. The copyright holder for this preprint (which was not certified by peer review) is the author/funder, who has granted bioRxiv a license to display the preprint in perpetuity. It is made available under aCC-BY-NC-ND 4.0 International license.

Target-Decoy MineR for determining the biological relevance of variables in noisy data sets. $-6 / 11$

\subsection{Principal component analysis}

The principal component analysis (PCA) TDM summary report was generated using the function fviz_pca_var() from the R package factoextra ${ }^{30}$.

\subsection{Data availability statement}

The data, R source, and scripts used in this study were deposited at the Bitbucket repository https://cesaremov@bitbucket.org/cesaremov/ Target-Decoy_MineR.git.

\section{Results}

We tested the Target-Decoy MineR (TDM) with synthetic and experimental metabolomics datasets.

\subsection{Processing synthetic datasets with defined pertur- bations}

The synthetic datasets were composed of two groups, 'wild type' (WT) and 'treatment' (TR) samples, with 500 variables. Ten sample replicates were simulated, and 50 variables in each group were perturbated ( 25 variables in WT and 25 values TR), with $0-, 0.5-, 1-$, and 2-log fold change perturbation (Fig. 1e-1).

The synthetic dataset demonstrates the inverse relation between the $\log F C$-fold perturbation and the TDM metric. The metric decreases as $\log F C$ increases (Fig. $\mathbf{1 i}-\mathbf{j}$ ). The metric approaches to zero for strong perturbations $\log F C \geq 1$. For each $\log F C$ perturbation, TDM produced different optimal FPR and BRp values. Optimum TDM scores are visualized in the FPR vs. BRp plots (Fig. 1k-1). The plots show both target and decoy points and their trend line. TDM on null perturbation data $(\log F C=0)$ finds the optimal values FPROpt $=0.49$ and BRpOpt $=0.45$, indicating the absence of discriminating variables. These values agree with the expectation that about $50 \%$ target and $50 \%$ decoy variables should be found in a dataset without two defined sample classes. Even top-ranked target variables are most likely unrelated to the sample class.

In stark contrast, if a strong perturbation was applied, such as $\log F C=2$, TDM obtained optimum values of FPROpt $=0.00$ and $B R p=0.99$. Top-ranked variables are strongly related to the sample class. In a biological experiment, these variables are likely to be of biological relevance because they contribute to the sample differentiation.

For the intermediate case, a mild perturbation with $\log F C=0.5$, TDM obtained values of FPROpt $=0.20$ and $B R p O p t=0.85$. Therefore, TDM detected non-random variables which are related to the sample class.

The synthetic datasets analysis demonstrated a predictable behavior of the Target-Decoy MineR results, depending on the variables' degree of perturbations.

\subsection{Processing experimental metabolomics data}

We used experimental metabolomics data of Drosophila melanogaster and Drosophila mojavensis fed on a high protein-to-sugar ratio (HPS) or equal protein-to-sugar ratio (EPS) diet (Fig. 2) ${ }^{19}$ to test the suitability of the Target-Decoy MineR for analyzing real-life data. For investigating the influence of technical noise and the number of features, the experimental data were either analyzed 'as is' or after additional pre-processing of the mass spectrometry data. Raw data contained 953 features, and denoised datasets 835 features (Fig. 2e-h) ${ }^{19}$.

The TDM analyses revealed false-positive rates (FPR) of 0.33 to 0.54 and biological relevance probabilities (BRp) of 0.50 to 0.54 for the 10 top-ranked variables of Drosophila melanogaster. Following their generalist and high carbohydrate diet adaptation, these values indicate the absence of a difference between the two sample classes HPS and EPS. Indeed, the two groups of flies did not exhibit a significant difference in their phenotype. In contrast, for Drosophila mojavensis, FPR of 0.00 and BRp values were of 0.99 to 1.00 for the top-10 variables, which indicates a stark metabolic difference between the two diets (Fig. 2m-p). This cactophilic fly species is highly specialized for its ecological niche and suffered developmental disorders during the experiment.

Thus, the biological effect of the treatments on the different fly species is reflected in the numerical scores of the Target-Decoy MineR.

\subsection{Statistical performance of the Target-Decoy MineR}

To investigate the statistical performance of the TargetDecoy MineR, we calculated the optimum metric and performed receiver operating curve (ROC), the area under the curve (AUC), sensitivity, and specificity calculations (Fig. 3). The AUC has been designated as the best single number indicator of the classification accuracy of Machine Learning algorithms ${ }^{28}$.

For synthetic datasets with non-null perturbations $(\log F C>0)$, AUC values higher than 0.6 indicate a working classifier. The AUC of the Target-Decoy MineR correlated positively with the degree of perturbation, $\log F C$. A $\log F C$ of $0,0.5,1$, and 2 resulted in an AUC of $0.56,0.75,0.94$ and 0.99 , respectively (Fig. $3 \mathbf{b}$ ). As expected, the building of a predictive model for sample classification failed for null-perturbation data $(\log F C=$ $0)$. For data with a mild perturbation $(\log F C=0.5)$ a fair model was obtained, and for $\log F C>1$, the TDM produced highly reliable models. Analogously, the sensitivity and specificity increased with the degree of the data perturbation (Fig. 3c-d).

Applying the Target-Decoy MineR to the Drosophila metabolomics data, an AUC of 0.28 was determined for Drosophila melanogaster and an AUC of 0.85 for 
bioRxiv preprint doi: https://doi.org/10.1101/2020.11.09.374181; this version posted November 10,2020 . The copyright holder for this preprint (which was not certified by peer review) is the author/funder, who has granted bioRxiv a license to display the preprint in perpetuity. It is made available under aCC-BY-NC-ND 4.0 International license.

Target-Decoy MineR for determining the biological relevance of variables in noisy data sets. $-7 / 11$

a

Raw data

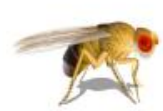

D. melanogaster b

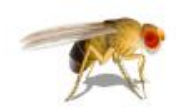

D. melanogaster denoised c

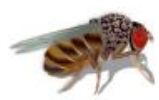

D. mojavensis d

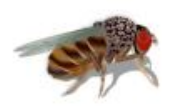

D. mojavensis denoised

e

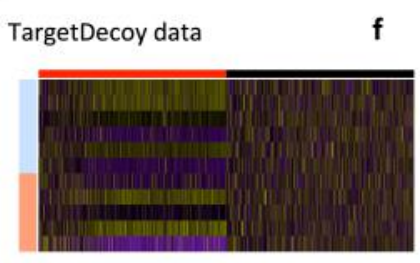

f

g

h

i TargetDecoy metric

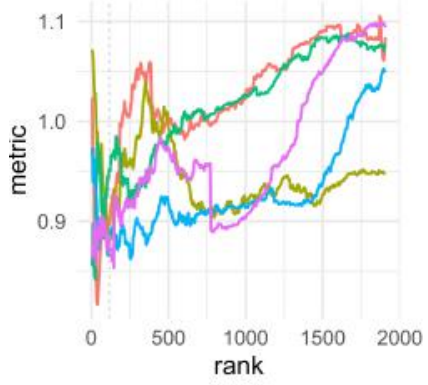

m Relevant variables

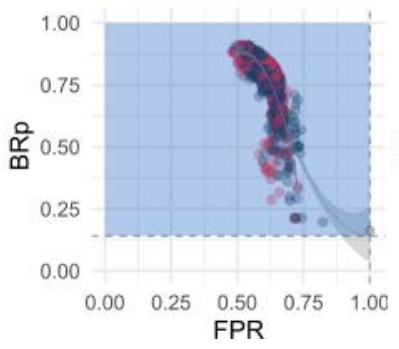

j

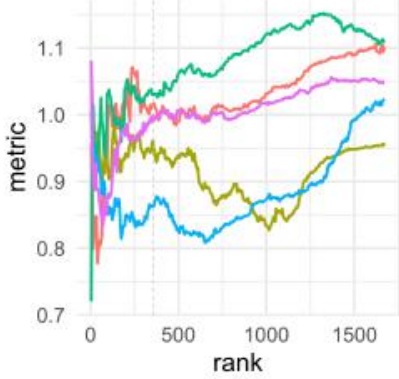

n

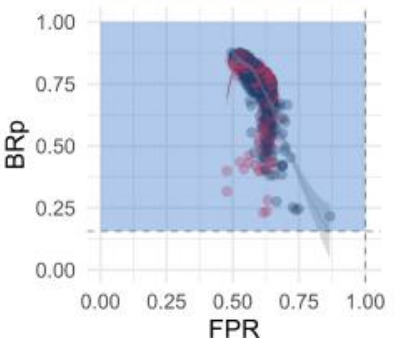

k

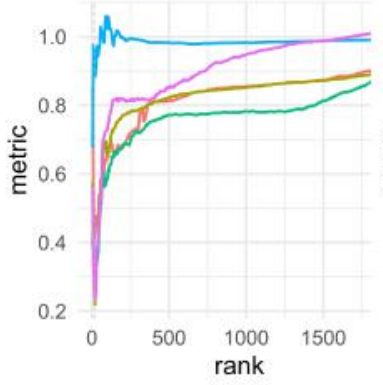

o

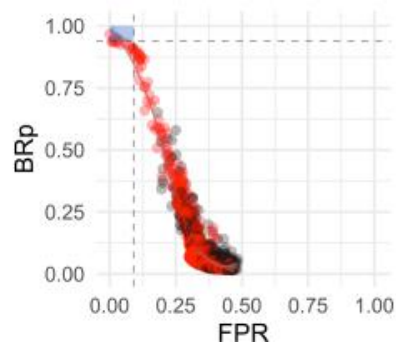

I

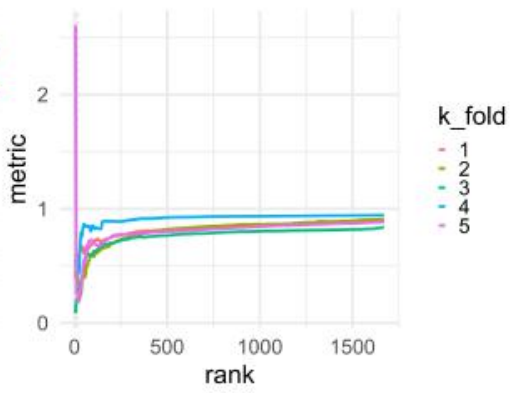

p

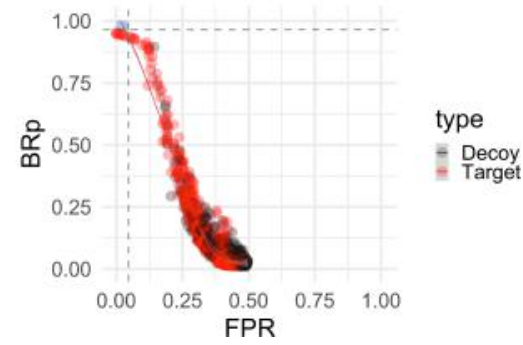

Figure 2. Identification of biologically relevant variables in metabolomics. Drosophila melanogaster and Drosophila mojavensis flies were fed different protein-sugar mixtures. a-h Target-decoy datasets obtained from raw and pre-processed (denoised) datasets. Raw datasets contain 953 targets + 953 Decoys. Denoised datasets contain 835 targets +835 decoys, $\mathbf{i}-\mathbf{l}$ Calculation of the optimum metric and rank, $\mathbf{m}-\mathbf{p}$ The Target-Decoy MineR plots indicate no significant effect of the different diet on the metabolism of Drosophila melanogaster, but a strong effect on the Drosophila mojavensis metabolism. Variables which are important for the classification are not clustered together with decoy variables. The algorithm was not affected by technical noise in raw data. 
bioRxiv preprint doi: https://doi.org/10.1101/2020.11.09.374181; this version posted November 10,2020 . The copyright holder for this preprint (which was not certified by peer review) is the author/funder, who has granted bioRxiv a license to display the preprint in perpetuity. It is made

Target-Decoy MineR for determining the biological relevance of variables in noisy data sets. $-8 / 11$

synthetic data

experimental data

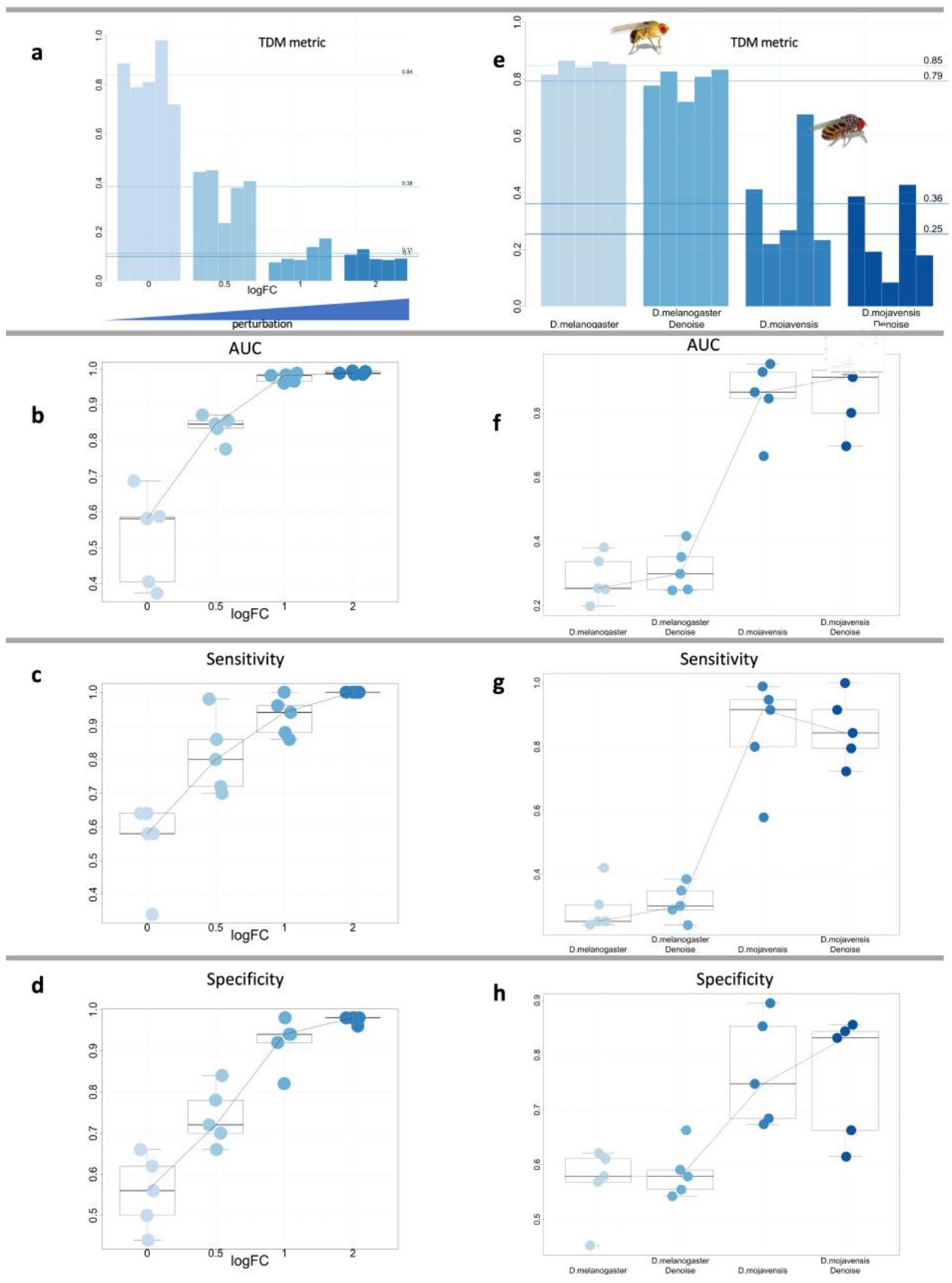

Figure 3. Target-Decoy MineR (TDM) evaluation of synthetic and experimental datasets. In the synthetic datasets, the TDM metric reduces as perturbation $(\log F C)$ increases. The Drosophila melanogaster datasets have a larger TDM metric than Drosophila mojavensis datasets, indicating a minor biological effect of diet on their metabolism. The area under the curve (AUC), sensitivity, and specificity increase with the system's perturbance. The TDM worked equally well for raw and denoised metabolomics data. 
bioRxiv preprint doi: https://doi.org/10.1101/2020.11.09.374181; this version posted November $10,2020$. The copyright holder for this preprint (which was not certified by peer review) is the author/funder, who has granted bioRxiv a license to display the preprint in perpetuity. It is made available under aCC-BY-NC-ND 4.0 International license.

Target-Decoy MineR for determining the biological relevance of variables in noisy data sets. $-9 / 11$

Drosophila mojavensis (Fig. 3f). Sensitivity and specificity measures behave accordingly (Fig. 3g-h). Again, the results indicate a high tolerance of Drosophila melanogaster flies against a change of their diet, equally tolerated both diets. In contrast, an accurate predictive model was created for Drosophila mojavensis, indicating that Drosophila mojavensis had metabolic changes.

\subsection{Robustness of the Target-Decoy MineR against noise}

We evaluated the TDM algorithm's sensitivity against noise comparing TDM results from using raw and denoised Drosophila data (Fig. 2 and 3). To denoise the data, we removed $m / z$ variables below a signal-to-noise threshold ${ }^{18}$. For Drosophila melanogaster, TDM obtained top-10 BRp scores ranging from 0.33 to 0.54 for raw data and top-10 BRp scores between 0.44 to 0.57 for denoised data. Both results are similar and indicate a negligible effect of diet on the metabolic profile of Drosophila melanogaster. Contrary, for Drosophila mojavensis, TDM determined BRp scores of 1.00 for the top-10 variables of both raw and denoised data, indicating a strong biological effect. These top-10 variables are $703.67,702.70$, $704.67,683.74,677.68,676.72,635.61,633.76,505.95$, and $502.71 \mathrm{~m} / \mathrm{z}$, and could be related to phospholipids with long and saturated fatty acids, which are associated with metabolic diseases and physiological desert life adaptation ${ }^{19}$. Using raw or pre-processed data had no significant impact on the outcome of the Target-Decoy MineR.

\section{Discussion}

The Target-Decoy MineR (TDM) finds the optimal falsepositive rate (FPR) and biological relevance probability (BRp) values by minimizing the metric of variable ranks (see Fig. 1). This metric compares a model built using 'informative variables' $m$ (top-ranked) to a model built upon 'uninformative' variables $n$. This metric can be thought of as an 'information loss' parameter. Thus, the selected model minimizes the 'sample class information loss' of variables ((Fig. $\mathbf{1}$ and $\mathbf{3 a}, \mathbf{b}))$.

For synthetic data, usable predictive models (AUC $=0.75)$ were created for even mildly perturbated $(\log F C$ $=0.5$ ) variables. Accurate classification models with an AUC higher than 0.9 were possible with perturbations $\log F C>1$. The AUC, sensitivity, and specificity increase with the system's perturbance for synthetic and experimental data (Fig. 3).

A Target-Decoy MineR model for raw and preprocessed metabolomics data of Drosophila mojavensis yielded 80 and 70 optimal variables, respectively. The correlation between raw and denoised BRp scores was 0.80 , with a $p$-value of 0.0001 . This result underlines the utility of the Target-Decoy MineR for unprocessed experimental data.

\section{Conclusion}

The addition of artificial data noise variables to experimental data further enhances the utility of machine learning strategies. The computed false-positive rates (FPR) and biological relevance probabilities (BRp) facilitate the separation between informative and uninformative variables. The Target-Decoy MineR (TDM) identifies biologically relevant variables from noisy data, even for poorly designed experiments with low statistical power, e.g., with few replicates and mild perturbation. The Target-Decoy MineR can be employed for experimental data classification and hypothesis testing.

Whereas the specificity-sensitivity dilemma is not entirely solved, now an informed decision for a reasonable cut-off for ranked variables is possible, using the FPR and BRp values. For example, in exploratory Omics studies, relatively high FPR and low BRp cut-offs could be acceptable, whereas, in detection biomarker search, strict criteria must be applied.

Strikingly, the Target-Decoy MineR is insensitive to data noise and applicable to pre-processed and raw experimental data.

The Target-Decoy MineR is easy to adapt to diverse types of massive data. An implementation of the TargetDecoy Miner as an R package is available at https:// bitbucket.org/cesaremov/targetdecoy_mining/, released under the GNU General Public License (GPL). The program is immediately applicable for tabular data.

\section{Acknowledgments}

The work was funded by the CONACyT-DFG grant 2016/277850 to RW. DCG acknowledges CONACyT for his scholarship. COV acknowledges the Cátedras CONACyT program.

Authors acknowledge IPICYT Supercomputing National Center (CNS) Thubat Kaal 2.0 computing resources provided to test Target-Decoy MineR under the project TKII-R2018-COV1.

\section{Author contributions}

DCG performed biological experiments. COV, DGC, and RW designed the study, analyzed the data, and wrote the manuscript. COV programmed and tested the Target-Decoy_MineR R package.

\section{Competing financial interests}

The authors declare no competing financial interests.

\section{References}

[1] Casey S. Greene, Jie Tan, Matthew Ung, Jason H. Moore, and Chao Cheng. Big data bioinformatics. J. Cell. Physiol., 229(12):1896-1900, December 2014. 
[2] Leo Breiman. Random Forests. Machine Learning, 45(1):5-32, 2001.

[3] Leo Breiman. Bagging Predictors. Machine Learning, 24(2):123-140, August 1996.

[4] Graham Williams. Data Mining with Rattle and R: The Art of Excavating Data for Knowledge Discovery (Use R!). Springer, 1st edition, August 2011. Published: Paperback.

[5] Leo Breiman, Jerome Friedman, Charles J. Stone, and R. A. Olshen. Classification and Regression Trees. Chapman and Hall/CRC, Boca Raton, 1st edition, January 1984.

[6] Corrado Gini. Variabilità e mutabilità. Reprinted in Memorie di metodologica statistica (Ed. Pizetti E, Salvemini, T). Rome: Libreria Eredi Virgilio Veschi, 1, 1912.

[7] Robert Winkler. Popper and the Omics. Frontiers in Plant Science, 7(195):1-3, 2016.

[8] Ramón Díaz-Uriarte and Sara Alvarez de Andrés. Gene selection and classification of microarray data using random forest. BMC Bioinformatics, 7:3, January 2006.

[9] Robert Winkler. An evolving computational platform for biological mass spectrometry: workflows, statistics and data mining with MASSyPup64. PeerJ, 3(e1401):1-34, 2015.

[10] Kim-Anh Lê Cao, Simon Boitard, and Philippe Besse. Sparse PLS discriminant analysis: biologically relevant feature selection and graphical displays for multiclass problems. BMC Bioinformatics, 12:253, June 2011.

[11] Matt R. Paul, Nicholas P. Levitt, David E. Moore, Patricia M. Watson, Robert C. Wilson, Chadrick E. Denlinger, Dennis K. Watson, and Paul E. Anderson. Multivariate models from rna-seq snvs yield candidate molecular targets for biomarker discovery: Snv-da. BMC Genomics, 17(1):263, Mar 2016.

[12] Robin Genuer, Jean-Michel Poggi, and Christine Tuleau-Malot. Variable selection using random forests. Pattern Recognition Letters, 31(14):22252236, October 2010.

[13] A. Hapfelmeier and K. Ulm. A new variable selection approach using Random Forests. Computational Statistics \& Data Analysis, 60:50-69, April 2013.
[14] Trevor Hastie, Robert Tibshirani, and Jerome Friedman. The Elements of Statistical Learning: Data Mining, Inference, and Prediction, Second Edition. Springer Series in Statistics. Springer-Verlag, New York, 2 edition, 2009.

[15] R. Kohavi. A study of cross-validation and bootstrap for accuracy estimation and model selection. Proceedings of the 14th international joint conference on Artificial intelligence - Volume 2, 2(0):1137-1143, 1995.

[16] Joshua E Elias and Steven P Gygi. Target-decoy search strategy for increased confidence in largescale protein identifications by mass spectrometry. Nat. Methods, 4(3):207-214, March 2007.

[17] Kyle D. Bemis, April Harry, Livia S. Eberlin, Christina Ferreira, Stephanie M. van de Ven, Parag Mallick, Mark Stolowitz, and Olga Vitek. Cardinal: an $\mathrm{R}$ package for statistical analysis of mass spectrometry-based imaging experiments. Bioinformatics, March 2015.

[18] Sebastian Gibb and Korbinian Strimmer. MALDIquant: a versatile $R$ package for the analysis of mass spectrometry data. Bioinformatics, 28(17):2270-2271, September 2012.

[19] Daniel Cázarez-García, Mariana Ramírez Loustalot-Laclette, Therese Ann Markow, and Robert Winkler. Lipidomic profiles of Drosophila melanogaster and cactophilic fly species: models of human metabolic diseases. Integrative Biology, 9(11):885-891, 2017.

[20] Luciano M. Matzkin, Sarah Johnson, Christopher Paight, Goran Bozinovic, and Therese A. Markow. Dietary Protein and Sugar Differentially Affect Development and Metabolic Pools in Ecologically Diverse Drosophila. J. Nutr., 141(6):1127-1133, June 2011.

[21] Tomáš Pluskal, Sandra Castillo, Alejandro VillarBriones, and Matej Orešič. MZmine 2: Modular framework for processing, visualizing, and analyzing mass spectrometry-based molecular profile data. BMC Bioinformatics, 11(1):395, July 2010.

[22] Victor Treviño, Irma-Luz Yañez-Garza, Carlos E Rodriguez-López, Rafael Urrea-López, Maria-Lourdes Garza-Rodriguez, Hugo-Alberto Barrera-Saldaña, José G Tamez-Peña, Robert Winkler, and Rocío-Isabel Díaz de-la Garza. GridMass: a fast two-dimensional feature detection method for LC/MS. Journal of Mass Spectrometry, 50(1):165-174, 2015. 
bioRxiv preprint doi: https://doi org/10.1101/202011.09 374181 this version posted November 10,2020 . The copyright holder for this preprint (which was not certified by peer review) is the author/funder, who has granted bioRxiv a license to display the preprint in perpetuity. It is made available under aCC-BY-NC-ND 4.0 International license.

Target-Decoy MineR for determining the biological relevance of variables in noisy data sets. $-11 / 11$

[23] R Core Team. R: A Language and Environment for Statistical Computing. R Foundation for Statistical Computing, Vienna, Austria, 2018.

[24] W. N. Venables and B. D. Ripley. Modern Applied Statistics with S. Springer, New York, fourth edition, 2002. ISBN 0-387-95457-0.

[25] Yoav Benjamini and Yosef Hochberg. Controlling the False Discovery Rate: A Practical and Powerful Approach to Multiple Testing. Journal of the Royal Statistical Society. Series B (Methodological), 57(1):289-300, 1995.

[26] Marvin N. Wright and Andreas Ziegler. ranger: A fast implementation of random forests for high dimensional data in $\mathrm{C}++$ and $\mathrm{R}$. Journal of Statistical Software, 77(1):1-17, 2017.

[27] David Meyer, Evgenia Dimitriadou, Kurt Hornik, Andreas Weingessel, and Friedrich Leisch. e1071: Misc Functions of the Department of Statistics, Probability Theory Group (Formerly: E1071), TU Wien, 2019. $\mathrm{R}$ package version 1.7-1.

[28] Andrew P. Bradley. The use of the area under the ROC curve in the evaluation of machine learning algorithms. Pattern Recognition, 30(7):1145-1159, July 1997.

[29] Xavier Robin, Natacha Turck, Alexandre Hainard, Natalia Tiberti, Frédérique Lisacek, Jean-Charles Sanchez, and Markus Müller. proc: an open-source package for $\mathrm{r}$ and $\mathrm{s}+$ to analyze and compare roc curves. BMC Bioinformatics, 12:77, 2011.

[30] Alboukadel Kassambara and Fabian Mundt. factoextra: Extract and Visualize the Results of Multivariate Data Analyses, 2017. R package version 1.0.5.999. 